\title{
Efectos de la violencia en la salud mental de estudiantes de enfermería
}

\author{
Effect of Violence in Nursing Students' Mental Health
}

Efeitos da violência na saúde mental de estudantes de enfermagem

\begin{abstract}
Jenny Mendoza-Rosado ${ }^{1 a}$
Yolanda Torres-Chávez ${ }^{1 \mathrm{~b}}$

Rina Mendoza-Rosado ${ }^{10}$

Johana Amaya Quisper ${ }^{1 d}$

Liz Delgado Pacohuanaco ${ }^{\text {1d }}$

Lizbeth Nina Salamanca ${ }^{1 \mathrm{~d}}$

Peggi Tuanama Fuchs ${ }^{\text {1d }}$
\end{abstract}

(iD) https://orcid.org/0000-0003-3002-7469

(iD) https://orcid.org/0000-0002-1586-9038

https://orcid.org/0000-0001-5054-3060

\section{Resumen}

Objetivo: Analizar los efectos de la violencia en la salud mental de los estudiantes de enfermería de la Universidad Nacional Jorge Basadre de Tacna, 2018- 2019. Material y métodos: Es un estudio cuantitativo, no experimental, descriptivo, de corte transversal. La técnica utilizada fue la encuesta; y el instrumento fue un cuestionario estructurado. La población fue de 176 estudiantes de primero a quinto año de enfermería matriculados en el 2019, quienes otorgaron su consentimiento informado Resultados: El $59 \%$ registró un VIF (negativo), evidenciando que no experimentaron violencia, el $34 \%$ presentó riesgo de violencia y el $7 \%$ registran VIF+ (positivo); es decir, sí experimentaron violencia. La mayor cantidad de estudiantes experimentó violencia psicológica (109 casos de los 176 estudiantes). Entre los efectos de violencia en la salud mental, se encontró depresión en los estudiantes de primero, segundo, tercero y quinto año con $50 \%, 37 \%, 33 \%$, y $38 \%$ respectivamente. En el 4to año el más alto efecto fue la conducta sexual inapropiada con $35 \%$; seguido de otro efecto significativo que fue intento de suicidio. Este último registró el más alto porcentaje en tercer año (28 \%). Conclusión: Los estudiantes de enfermería de la Universidad Nacional Jorge Basadre Grohmann experimentaron algún tipo de violencia, los efectos en su salud mental fueron la depresión, la conducta sexual inapropiada e intento de suicidio.

Palabras claves: estudiantes de enfermería, violencia, salud mental

\section{Abstract}

Objective: To analyze the effects of violence on the mental health of nursing students at the Jorge Basadre National University of Tacna, 2018-2019. Material and methods: It is a quantitative, non-experimental, descriptive, cross-sectional study. The technique used was the survey; and the instrument was a structured questionnaire. The population was 176 students from 1 st to 5 th year of nursing enrolled in 2019, who granted their informed consent. Results: $59 \%$ registered a VIF - (negative), evidencing that they did not experience violence, $34 \%$ presented risk of violence and $7 \%$ registered VIF + (positive); that is, they did experience violence. The

\footnotetext{
${ }^{1}$ Escuela Profesional de Enfermería, Universidad Nacional Jorge Basadre Grohamn. Tacna, Perú

a Doctora en Ciencias: Enfermería

${ }^{\mathrm{b}}$ Magister en Docencia universitaria y Gestión Educativa

' Maestra en Gestión Pública

${ }^{d}$ Estudiantes de Enfermería
} 
largest number of students experienced psychological violence (109 cases out of 176 students). Among the effects of violence on mental health, depression was found in students of $1 \mathrm{st}, 2 \mathrm{nd}$, 3rd and 5th with $50 \%, 37 \%, 33 \%$, and $38 \%$ respectively. In the 4th year the highest effect was inappropriate sexual behavior with $35 \%$; followed by another significant effect which was suicide attempt. In the 4th year the highest effect was inappropriate sexual behavior with $35 \%$; followed by another significant effect that is suicide attempt, registering a higher percentage in the 3rd year (28\%). Conclusion: Nursing students at Jorge Basadre Grohmann University experienced some type of violence, the effects on their mental health were depression, inappropriate sexual behavior and suicide attempt.

Keywords: nursing students, mental health, violence

\section{Resumo}

Objetivo: Analisar os efeitos da violência na saúde mental de estudantes de enfermagem da Universidade Nacional Jorge Basadre de Tacna, 2018 a 2019. Material e métodos: trata-se de um estudo quantitativo, não experimental, descritivo e transversal. A técnica utilizada foi a pesquisa; e o instrumento foi um questionário estruturado. A população foi de 176 alunos do primeiro ao quinto ano do curso de enfermagem matriculados em 2019, que deram o seu consentimento informado. Resultados: $59 \%$ registraram VIF - (negativo), mostrando que não sofreram violência, 34\% apresentaram risco de violência e $7 \%$ registram VIF + (positivo); isto é, eles sofreram violência. O maior número de alunos sofreu violência psicológica (109 casos de 176 alunos). Entre os efeitos da violência na saúde mental, a depressão foi identificada em alunos do primeiro, segundo, terceiro e quinto ano com 50\%, 37\%, 33\% e 38\% respectivamente. No quarto ano, o maior efeito foi o comportamento sexual inadequado com $35 \%$; seguido por outro efeito significativo que foi a tentativa de suicídio. Este último registrou o maior percentual no terceiro ano $(28 \%)$. Conclusão: Os alunos de enfermagem da Universidade Nacional Jorge Basadre Grohmann vivenciaram algum tipo de violência, os efeitos em sua saúde mental foram depressão, comportamento sexual inadequado e tentativa de suicídio.

Palavras-chave: estudantes de enfermagem, violência, saúde mental

\section{Introducción}

En los últimos años la salud mental ha cobrado gran importancia, especialmente porque las tasas de violencia se han incrementado a tal punto que se han diseñado políticas nacionales e internacionales para proteger a la población vulnerable de la violencia. La universidad quien lleva a cabo la formación académica, tiene la gran responsabilidad de formar integralmente al futuro profesional, pero a su vez fomentar el desarrollo de sus competencias. La investigación partió de una observación y diálogo con estudiantes de enfermería, quienes reportaron actitudes de susceptibilidad, como estar a la defensiva, baja autoestima, incumplimiento de tareas, no aportar para los trabajos en grupo, no socializar fácilmente y en su mayoría refieren no llevarse bien con sus padres. Otros casos provienen de hogares disfuncionales donde primaron los gritos, críticas, insultos, comparaciones, amenazas, coacciones e insultos con palabras soeces. El estudio, analizó los efectos de la violencia en la salud mental de los estudiantes en las diversas dimensiones. Esta Investigación responde a las necesidades y prioridades de investigación en la Región Tacna, a nivel regional, la violencia es una de las prioridades de investigación en salud para el quinquenio 2015 - 2021, también en el área de salud mental con el código 1.14. violencia intrafamiliar y trastornos psicoafectivos. ${ }^{13} \mathrm{~A}$ nivel de la UNJBG se encuentra en la línea de investigación servicios de salud y calidad de vida. 
La ONU reveló que durante el año 2015 en la región Tacna, el 52,1 \% de mujeres padecieron alguna forma de violencia psicológica o verbal. ${ }^{1}$ La OMS define la violencia como "el uso deliberado de la fuerza física o el poder, ya sea en grado de amenaza o efectivo, contra uno mismo, otra persona o un grupo o comunidad, que cause o tenga muchas posibilidades de causar lesiones, muerte, daños psicológicos, trastornos del desarrollo o privaciones". ${ }^{3}$ La violencia fue abordada desde enfoques psicológico, antropológico, sociológico, biológico y conflicto social; así como desde teorías de la violencia $^{14,15,16}$ (Figura 1).

\section{Figura 1}

\section{Enfoques teóricos de la violencia}

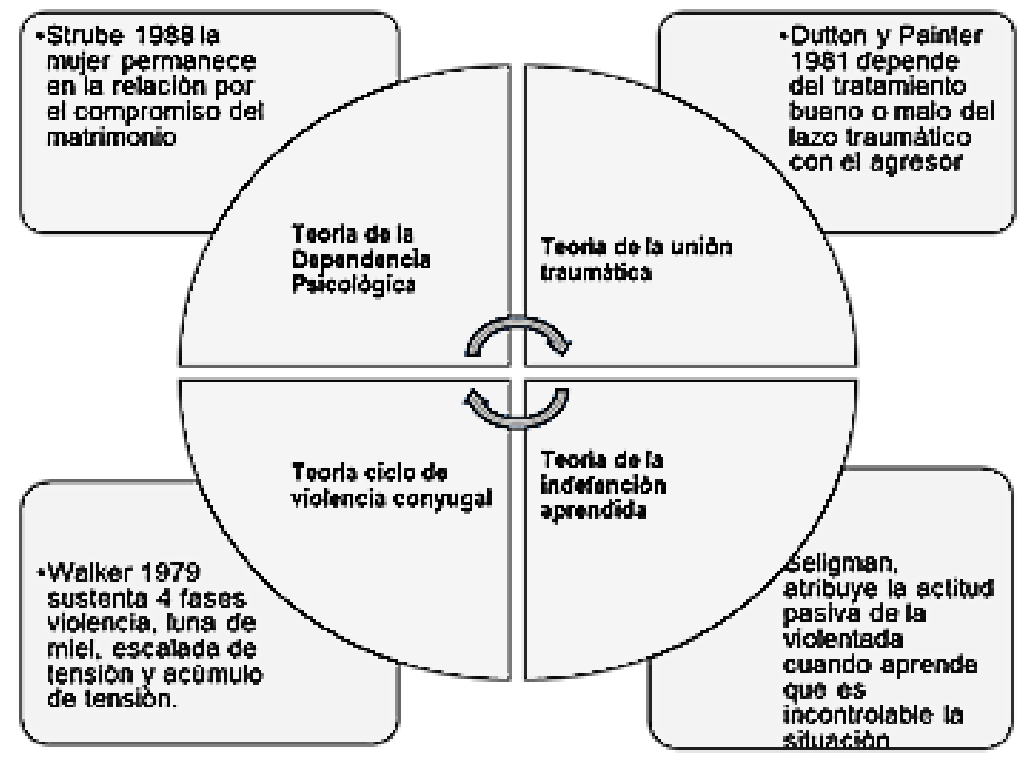

Nota. Elaborado por Dr. Jenny Mendoza Rosado.

Cada enfoque teórico, aporta las bases para la comprensión del fenómeno de estudio llamado violencia.

Para comprender la violencia es necesario conocer los enfoques y también las caracterizaciones de los diversos tipos de violencia. ${ }^{5,17} \mathrm{~A}$ continuación nos referiremos a las diversas manifestaciones de violencia.

\section{La violencia física}

Es la acción o conducta que causa daño a la integridad corporal o a la salud de una persona o más del grupo familiar utilizando la fuerza física. La evidencia de la violencia física son las lesiones en el cuerpo, que se encuentran en un rango de agresiones muy amplio; desde un empujón, bofetadas, tracción de cabellos, intentos de estrangulación, torceduras de brazo, golpes de puño, puntapiés, golpes con objetos, quemaduras, agresión con armas de fuego o punzocortantes, hasta lesiones graves con secuelas permanentes o la muerte misma.

La violencia psicológica es toda agresión entre las personas cuando una o más de ellas arremeten de manera verbal a otra u otras personas, ocasionando algún tipo de daño a nivel psicológico o emocional en las personas agredidas. Se enfoca en la emisión de frases descalificadoras y humillantes que buscan desvalorizar al otro, con gritos, crítica, comparaciones, malos gestos, amenazas, coacción, someterle a aislamiento y otras formas degradantes de reclusión. 


\section{La violencia sexual}

Comprende los delitos que se cometen contra una persona sin su consentimiento o bajo coacción de violación de la libertad sexual, proxenetismo y ofensas al pudor público a los que se refieren en el Código Penal Peruano, Ley n. ${ }^{\circ} 30364$ y en la Constitución Política del Perú. La violencia sexual incluye actos que involucran penetración o contacto físico alguno, insinuaciones verbales o físicas de carácter sexual, así como ser expuesto a material pornográfico y aquellos que vulneren el derecho a las personas de decidir voluntariamente. $^{21}$

\section{La violencia social ambiental}

Es la acumulación de muchas violencias personales que produce un estado social generalizado o restringido de falta de respeto a la naturaleza, la vida y la libertad de los seres, violencia más o menos sutil, indirecta medioambientales, económicos, políticos 0 sociales, o directa, que ejercen sobre nosotros, el dogmatismo y la intolerancia ideológica, así como en cualquier violación colectiva de los derechos humanos. ${ }^{27}$

\section{La violencia económica}

Es la acción u omisión que se dirige a ocasionar un menoscabo en los recursos

\section{Figura 2}

Componentes de la salud mental

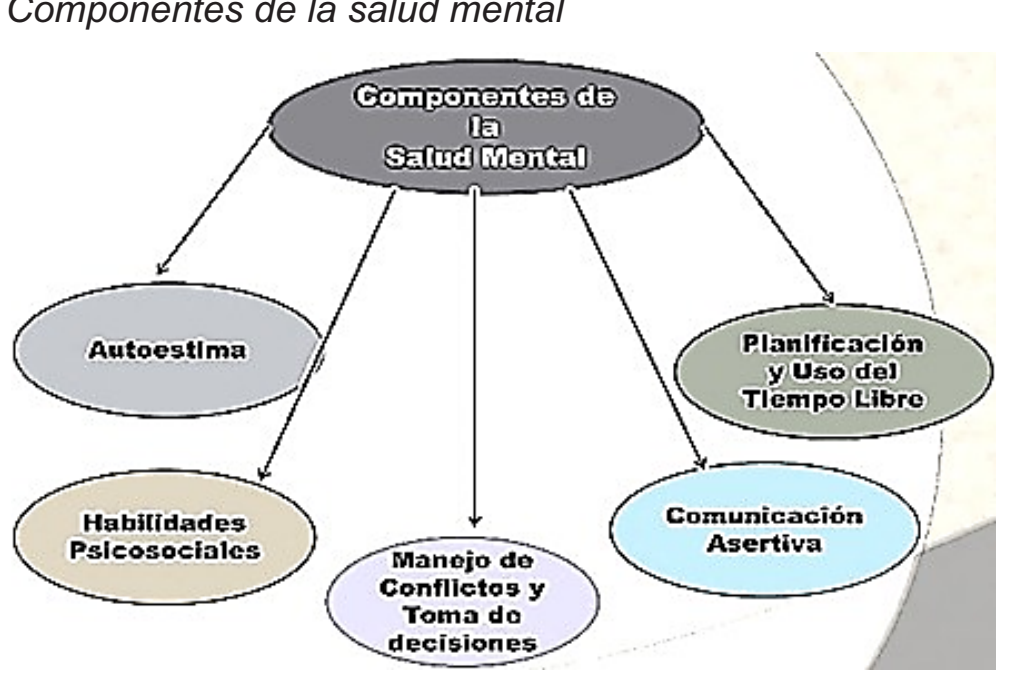

Nota. Elaborado por las autoras. económicos o patrimoniales de cualquier persona, se da cuando uno de los miembros de la familia usa el poder económico para provocar un daño a otro privándole de satisfacer sus necesidades o de los medios indispensables para vivir una vida digna; así como la evasión del cumplimiento de sus obligaciones alimentarias.

\section{La negligencia}

Es la falta de atención que requiere un niño al privarlo de cuidados. Consiste en no prestarle apoyo emocional, descuido y desatención a las necesidades básicas, médicas, o educacionales, así como exponerlo a violencia doméstica.

\section{Transtornos de salud mental}

La salud mental según la OMS es un estado de bienestar donde la persona es consciente de sus propias capacidades, puede afrontar las tensiones normales de la vida, puede trabajar de forma productiva y fructífera y es capaz de hacer una contribución a su comunidad. La buena salud mental está relacionada con el bienestar mental y psicológico. ${ }^{2}$ Incluye el bienestar emocional, psicológico y social, afecta la forma en que pensamos, sentimos y actuamos cuando enfrentamos la vida y cómo manejamos el estrés, nos relacionamos con los demás y tomamos decisiones. ${ }^{18}$ 
La Organización Mundial de la Salud (OMS 2019), en su publicación virtual, menciona que los trastornos mentales representan el $16 \%$ de la carga mundial de enfermedades y lesiones en personas de 10 a 19 años. La mitad de los trastornos mentales comienzan a los 14 años, pero la mayoría de los casos no se detectan ni se tratan. El suicidio es la tercera causa de muerte en jóvenes de 15 a 19 años. Además, es la segunda causa principal de defunción en el grupo etario de 15 a 29 años.

\section{Los trastornos de salud mental ${ }^{16}$}

Cada año todos los países en materia de salud, establecen las diez primeras enfermedades o trastornos de la salud mental, entre las más frecuentes tenemos:

- La depresión es el trastorno mental que se caracteriza por una profunda tristeza, decaimiento anímico, baja autoestima, pérdida de interés, de placer y bajo nivel de energía.

- La psicosis está caracterizada por distorsiones del pensamiento y de la percepción, así como también por un conjunto de emociones inapropiadas 0 limitadas. Puede presentar lenguaje incoherente o ilógico. También, se pueden presentar alucinaciones.

- La epilepsia o crisis epilépticas es una enfermedad crónica, caracterizada por convulsiones recurrentes no provocadas. Las crisis son causadas por descargas anormales en el cerebro y pueden ser de diferentes formas.

- Los trastornos por uso de alcohol es la dependencia de alcohol es un conjunto de fenómenos fisiológicos, conductuales y cognitivos. A menudo está asociado con consecuencias sociales (por ejemplo, problemas familiares o problemas en el trabajo), incluye la intoxicación aguda por alcohol, consumo perjudicial, síndrome de dependencia de alcohol y el estado de abstinencia de alcohol.

- Los trastornos por uso de drogas es la dependencia de drogas. Es un conjunto de fenómenos fisiológicos, conductuales y cognitivos en los cuales el consumo de drogas asume una prioridad mucho más alta para un determinado individuo en comparación con otras conductas.

- El suicidio es el acto deliberado de quitarse la vida. La autolesión es un término más amplio que concierne al envenenamiento o lesión intencional autoinfligida, la cual puede o no tener un objetivo o resultado fatal.

En enfermería, la teoría psicodinámica de Hildegarde Peplau fundamenta su utilidad en la capacidad de entender la propia conducta y ayudar a otras personas a identificar cuáles son sus dificultades, para aplicar principios de las relaciones humanas y abordar problemas que surgen en cualquier nivel de experiencia; describe que en la interrelación enfermera paciente se desarrollan cuatro fases.

La primera es la orientación. Aquí el paciente tiene una necesidad insatisfecha y precisa apoyo profesional del enfermero/a, quien ayuda al paciente a reconocer y entender su problema y determinar qué tipo de ayuda necesita. Esta etapa de dependencia se caracteriza por la vulnerabilidad de la persona, y es en este punto donde la enfermera tiene la gran tarea de cambiar la visión de víctima a persona que irá descubriendo su potencial.

La segunda, la identificación. En esta fase, el paciente se identifica con los que pueden ayudarle (relación), y es capaz de comprender la naturaleza de sus problemas, así como las oportunidades de intervenir en ellos, conjuntamente con el enfermero. Este es el inicio de una etapa de interdependencia, donde la persona reconoce que no es carente en su totalidad, va entendiendo la situación, y va descubriendo sus potencialidades y nuevas formas de abordar su situación.

La tercera etapa es la explotación. Aquí el paciente intenta obtener todo lo que se le ofrece a través de la relación de ayuda, apoyo y asesoramiento, poniendo en acción el máximo potencial que lleva dentro de sí la persona, para enfrentar sus problemas o necesidades, bajo el asesoramiento del enfermero. Es una etapa de franca interdependencia para avanzar hacia el próximo nivel, donde la persona logra tomar las riendas de su vida, retome la capacidad de decidir y sacar lo mejor de la adversidad. 
La resolución, la cuarta etapa, donde el paciente se libera de su situación con la ayuda y orientación del enfermero, para experimentar su independencia y auto cuidarse cuando se estabilice y retorne a su hogar. En esta etapa la persona recobra su capacidad de enrumbar su vida y tomar decisiones con una nueva actitud, sobre su situación enfrentada, y las que vendrán.

Esta teoría permite que los profesionales de enfermería dejen de centrarse en la atención biologicista de la enfermedad y puedan explorar los sentimientos y conductas propias del otro, de igual forma le permite al ser cuidado participar en las intervenciones de enfermería para cuidarlo.

\section{Material y métodos}

Estudio cuantitativo, de tipo no experimental, básico y descriptivo, de corte transversal, usando como técnica la encuesta y como instrumento el cuestionario del Ministerio de Salud modificado y adaptado por los autores para estudiantes universitarios, y evaluar la violencia en sus diversas dimensiones con indicadores binomiales, tales como 1) violencia física 5 items, 2) violencia sexual 6 items, 3) violencia psicológica 10 items, 4) violencia social-ambiental 6 items, 5) violencia económica 4 items y 6) negligencia 5 items Además, para evaluar salud mental a través de tamizaje de ansiedad, 7) depresión 18 items, 8) psicosis 4 items, 9) adicciones 6 items, 10) evidencia de violencia física 8 items, 11) violencia psicológica 16 items, 12) violencia sexual 4 items, 13) negligencia 7 items. La población participante fue de 176 estudiantes de 1 ro a 5to año de enfermería matriculados en el 2019 y que otorgaron su consentimiento informado.

\section{Resultados}

El $59 \%$ de estudiantes no experimentó violencia intrafamiliar (VIF-), el 34 $\%$ tenía riesgo de violencia, y el $7 \%$ sí experimentó violencia (VIF+) (Tabla y Figura 1).

La mayor cantidad de estudiantes experimentó violencia psicológica (109 casos), el 3er año registra los más altos porcentajes en los diversos tipos de violencia: física $59 \%$, psicológica $41 \%$, sexual $33 \%$, socio ambiental $38 \%$, económico $33 \%$ y por negligencia $50 \%$, en comparación con los demás años (2do, 4to y 5to. año) que registran porcentajes no menos significativos igualmente en todos los tipos de violencia (Tabla y Figura 2).

Los resultados de los efectos de la violencia en la salud mental en estudiantes fueron: $50 \%$ de los estudiantes fue afectado por depresión en primer año, la misma afección se registró en un $37 \%$ de los estudiantes de segundo año y en un $38 \%$ de los estudiantes de quinto año. Otros efectos resaltantes fueron: conducta sexual inapropiada en un $35 \%$ de los estudiantes de cuarto año e intento de suicidio en el $11 \%$ de los estudiantes de primer año. Este último se registró también en un $9 \%$ de los estudiantes de segundo, $28 \%$ de los estudiantes de tercero, $25 \%$ de los estudiantes de cuarto y en un $24 \%$ de los estudiantes de quinto año.

\section{Tabla 1}

Tipo de violencia en estudiantes de enfermería

\begin{tabular}{lllllllll}
\hline Violencia & \multicolumn{2}{c}{ VIF +} & \multicolumn{2}{c}{ VIF - } & \multicolumn{2}{c}{ VIF Riesgo } & \multicolumn{2}{c}{ Total } \\
\cline { 2 - 9 } Sección & $n$ & $\%$ & $n$ & $\%$ & $n$ & $\%$ & $n$ & $\%$ \\
\hline 1ro & 3 & 2 & 23 & 13 & 6 & 3 & 32 & 18 \\
2do & 2 & 1 & 16 & 9 & 9 & 5 & 27 & 15 \\
3ro & 4 & 2 & 21 & 12 & 24 & 15 & 49 & 28 \\
4to & 0 & 0 & 27 & 15 & 6 & 3 & 33 & 19 \\
5to & 4 & 2 & 17 & 10 & 14 & 8 & 35 & 20 \\
\hline Total & 13 & 7 & 104 & 59 & 59 & 34 & 176 & $100 \%$
\end{tabular}




\section{Figura 1}

Tipo de violencia en estudiantes de enfermería

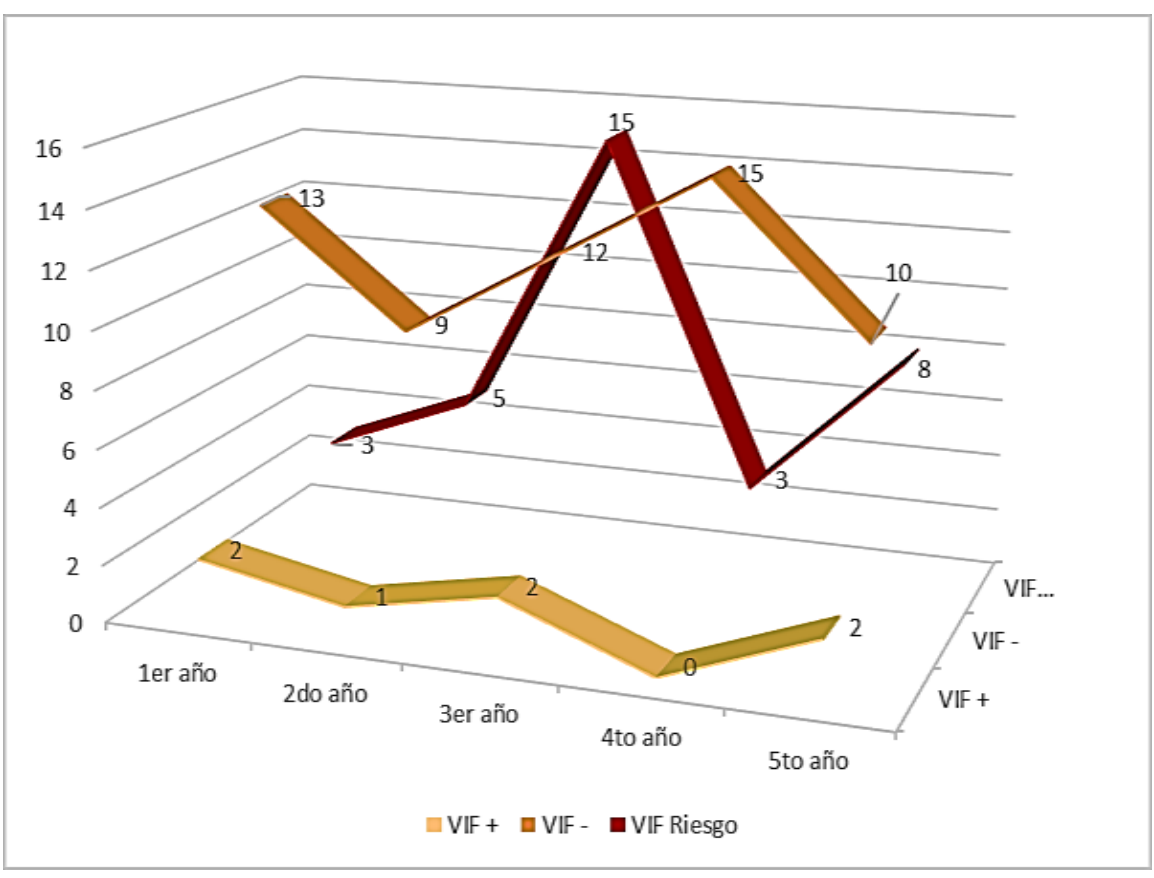

\section{Tabla 2}

Tipos de violencia detectada en estudiantes de enfermería

\begin{tabular}{|c|c|c|c|c|c|c|c|c|c|c|c|c|}
\hline \multirow{2}{*}{$\begin{array}{l}\text { Tipos de } \\
\text { violencia }\end{array}$} & \multicolumn{2}{|c|}{ Físico } & \multicolumn{2}{|c|}{ Psicológico } & \multicolumn{2}{|c|}{ Sexual } & \multicolumn{2}{|c|}{ Socio.Am } & \multicolumn{2}{|c|}{ Económ } & \multicolumn{2}{|c|}{ Negligencia } \\
\hline & $n$ & $\%$ & $n$ & $\%$ & $n$ & $\%$ & $n$ & $\%$ & $n$ & $\%$ & $n^{\circ}$ & $\%$ \\
\hline 1ro & 5 & 9 & 20 & 18 & 2 & 3 & 0 & 0 & 0 & 0 & 4 & 8 \\
\hline 2do & 9 & 16 & 20 & 18 & 15 & 24 & 9 & 31 & 7 & 26 & 12 & 23 \\
\hline 3ro & 33 & 59 & 44 & 41 & 20 & 33 & 11 & 38 & 9 & 33 & 26 & 50 \\
\hline 4to & 4 & 7 & 8 & 7 & 15 & 24 & 4 & 14 & 5 & 19 & 6 & 11 \\
\hline 5to & 5 & 9 & 17 & 16 & 10 & 16 & 5 & 17 & 6 & 22 & 4 & 8 \\
\hline Total & 56 & 100 & 109 & 100 & 62 & 100 & 29 & 100 & 27 & 100 & 52 & 100 \\
\hline
\end{tabular}




\section{Figura 2}

Tipos de violencia detectada en estudiantes de enfermería

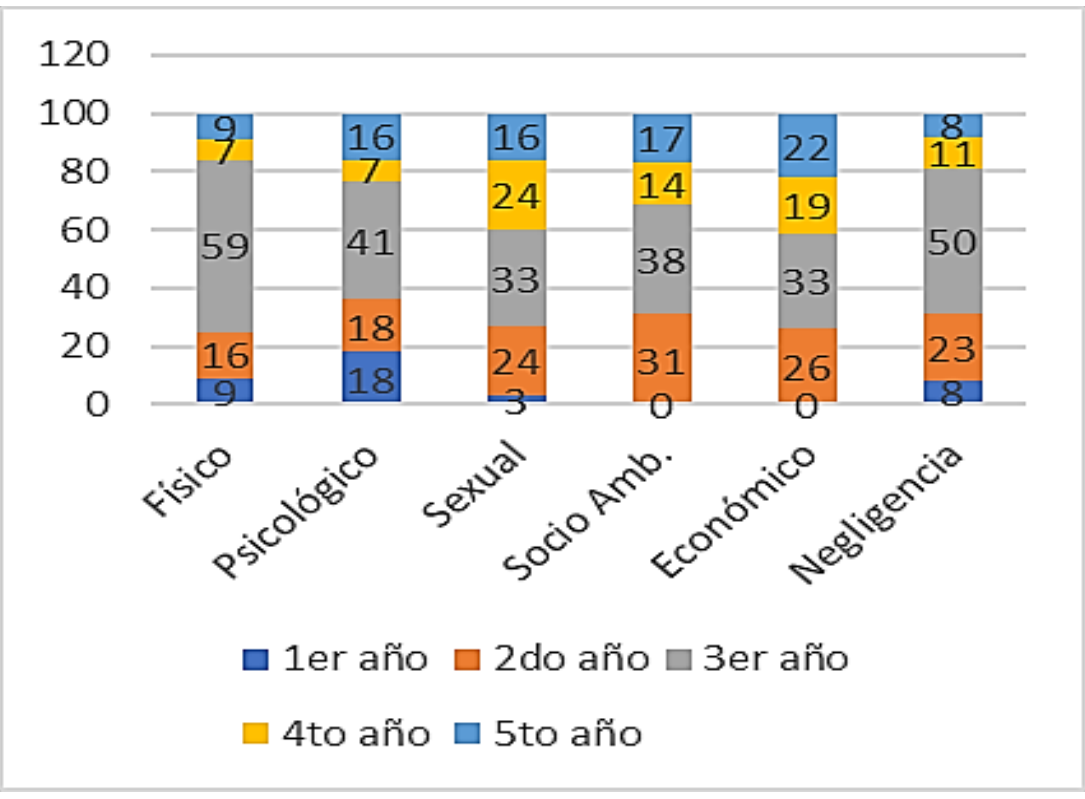

\section{Tabla 3}

Efectos de la violencia en la salud mental de estudiantes de enfermería

\begin{tabular}{lllllllllll}
\hline \multirow{2}{*}{ Efectos } & Años & \multicolumn{2}{c}{ 1ro } & \multicolumn{2}{c}{ 2do } & \multicolumn{3}{c}{3 ro } & \multicolumn{3}{c}{ 4to } & \multicolumn{2}{c}{5 to } \\
\cline { 2 - 12 } & $n$ & $\%$ & $n$ & $\%$ & $n$ & $\%$ & $n$ & $\%$ & $n$ & $\%$ \\
\hline Angustia & 6 & 11 & 0 & 0 & 1 & 1 & 0 & 0 & 0 & 0 \\
Depresión & 28 & 50 & 20 & 37 & 26 & 33 & 5 & 25 & 8 & 38 \\
Psicosis Riesgo & 0 & 0 & 18 & 33 & 9 & 11 & 0 & 0 & 1 & 5 \\
*Adicciones & 5 & 9 & 3 & 6 & 13 & 16 & 3 & 15 & 3 & 13 \\
Intento Suicidio & 6 & 11 & 5 & 9 & 22 & 28 & 5 & 25 & 5 & 24 \\
Tartamudeo & 9 & 16 & 2 & 4 & 2 & 3 & 0 & 0 & 2 & 10 \\
Conducta sexual & 2 & 3 & 6 & 11 & 6 & 8 & 7 & 35 & 2 & 10 \\
inapropiada & & & & & & & & & & \\
\hline Total & 56 & 100 & 54 & 100 & 79 & 100 & 20 & 100 & 21 & 100 \\
\hline
\end{tabular}


Figura 3

Efectos de la violencia en la salud mental de estudiantes de enfermería

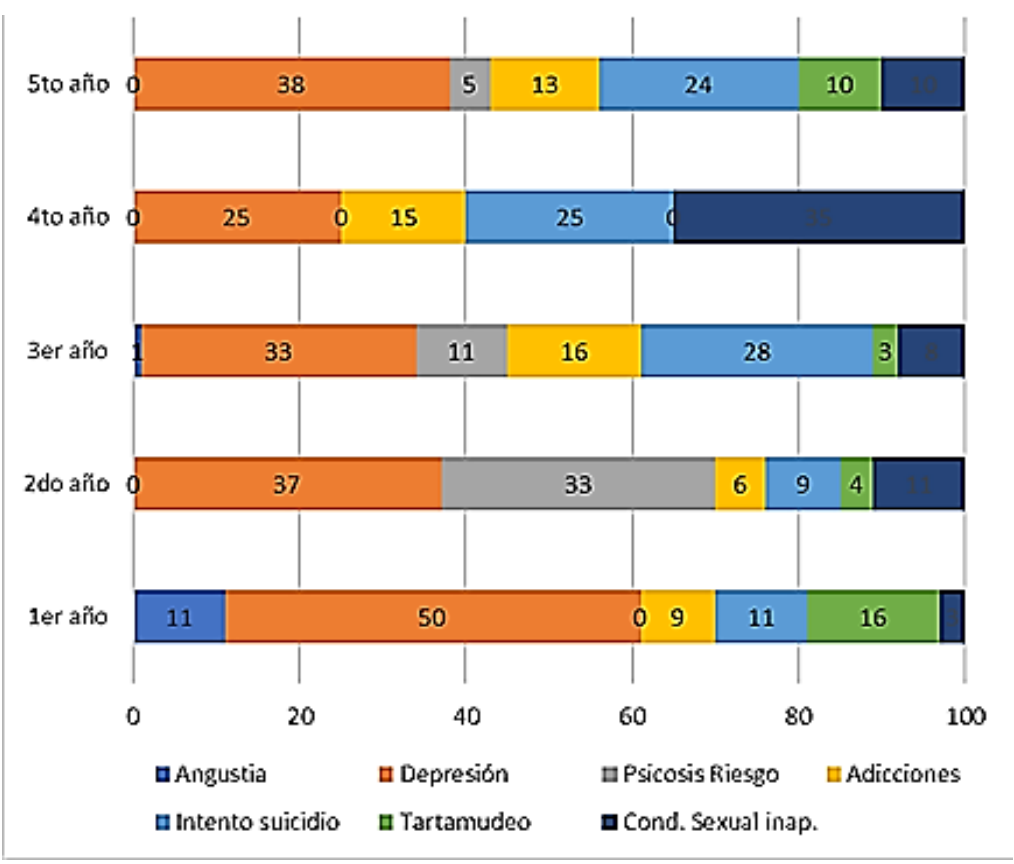

\section{Discusión}

Los resultados son reveladores, la Tabla 1 muestra que un considerable $59 \%$ tiene un VIF - (negativo), evidencia que la mayoría no experimentan violencia, seguido de un importante $34 \%$ que tienen riesgo de violencia y el $7 \%$ de estudiantes registran un VIF+ (positivo); es decir, sí experimentaron violencia. En ese aspecto el 3er año (15\%) fue el grupo que registró el más alto porcentaje de VIF en riesgo, seguido por el 5 to año (8 \%); y el 2 do año (5\%) y, finalmente, el 1 ro y 4 to año con el $3 \%$. Aunque el porcentaje es bajo, no deja de ser importante para la salud mental, así el $2 \%$ registraron VIF + en estudiantes de 1ro, 3ro y 5 to año, seguido del $1 \%$ en estudiantes de $2 \mathrm{do}$ año. Quienes registraron riesgo de violencia como aquellos que registraron violencia positiva intrafamiliar, tienen menoscabado su sistema de defensa física y psicológica. Estos resultados coinciden con Chávez Ayala, ${ }^{4}$ en cuanto al contexto familiar, todas las variables de salud mental fueron mediadoras por violencia moderada. Espinoza Godoy ${ }^{6}$ quien refiere que aunque el tema de la violencia que ejercen algunos padres sobre sus hijos se aborda muy poco, es importante que los progenitores conozcan que sus efectos pueden ser devastadores, ya que los niños o jovencitos que son sometidos continuamente a situaciones de agresiones presentan un debilitamiento gradual de sus defensas, tanto físicas como psicológicas. También, les infunde la sensación de que no son adecuados y de que no merecen contar con personas, tener cosas o plantearse metas valiosas, lo que les conduce a un fracaso en muchos aspectos de sus vidas. Paralelamente a esto, una de las consecuencias más nocivas de la violencia es que los hijos que aprenden modelos de relación violentos en su hogar tienden a reproducirlos; es decir, a llevarlos a la práctica en sus futuras relaciones. Varillas Suárez $z^{7}$ afirma que, para que la conducta violenta sea posible, tiene que darse un cierto desequilibrio de poder, que puede estar definido culturalmente o por el contexto o producido por maniobras 
interpersonales de control de la relación. Por su parte, la Federación Mundial de la Salud Mental WFMH resaltó el derecho de los jóvenes a crecer "saludables, felices y resilientes".

La Tabla 2 muestra la mayor cantidad de estudiantes con violencia psicológica (109 casos), el 3er año registró los más altos porcentajes en los diversos tipos de violencia: físico $59 \%$, psicológico $41 \%$, sexual $33 \%$, socio ambiental $38 \%$, económico $33 \%$ y por negligencia $50 \%$, en comparación con los demás años (2do, 4to y 5to. año), los cuales registran porcentajes no menos significativos; en todos los tipos de violencia. Se observó que en 1 er año los estudiantes sufrían violencia física $9 \%$, psicológica $18 \%$, sexual $3 \%$ y por negligencia $8 \%$, registran $0 \%$ en violencia socio ambiental y económica. La OMS manifiesta al respecto que la violencia (incluidos los malos tratos y la intimidación) y los problemas socioeconómicos son riesgos reconocidos para la salud mental. ${ }^{2}$ Espinoza Godoy ${ }^{6}$ dice que jovencitos que son sometidos continuamente a situaciones de agresiones presentan un debilitamiento gradual de sus defensas, tanto físicas como psicológicas. Igualmente, Varillas Suarez, ${ }^{7}$ refiere que la violencia siempre es una forma de ejercicio del poder mediante el empleo de la fuerza (ya sea física, psicológica, económica, etc.) e implica la existencia de un "arriba y un abajo", reales o simbólicos.

En la Tabla 3, entre los efectos de la violencia en la salud mental de los estudiantes, observamos que los porcentajes más altos los registró la depresión en 1 ro con el $50 \%$, en 2 do con $37 \%$, en 3 ro con $33 \%$, y en 5 to con $38 \%$. En el 4to año el más alto efecto fue la conducta sexual inapropiada con $35 \%$; seguido de otro efecto significativo que fue el intento de suicidio en 1 ro el $11 \%$, en 2 do el $9 \%$, en 3 ro el $28 \%$ en 4 to el $25 \%$ y en 5 to el $24 \%$. La OMS señala que los trastornos más frecuentes son los emocionales como depresión, ansiedad e irritabilidad, suicidio y autolesiones, trastornos alimentarios y de comportamiento, psicosis y conductas de riesgo. Las consecuencias de no abordar los trastornos mentales de los adolescentes se extienden a la edad adulta, lo que afecta la salud física y mental y limita las oportunidades de llevar una vida satisfactoria como adultos. ${ }^{2}$ Estas informaciones coincide con la Federación Mundial de la Salud Mental WFMH, que señala la importancia de cuidar la salud mental de los jóvenes, donde la depresión es la primera causa de carga de morbilidad entre los jóvenes y el suicidio, la segunda causa de muerte entre los 15 y 29 años. ${ }^{3}$ Chávez-Ayala señala las variables de salud mental que parcialmente actuaron como mediadoras fueron ideación suicida (35,9\%), depresión (19,2\%), intento de suicidio $(17,7 \%)$, autoestima emocional $(6,2 \%)$ y autoestima escolar $(3,4 \%)$, por la violencia moderada en la familia, y autoestima emocional $(17,5 \%)$ por rechazo social en la escuela e ideación suicida $(8,1 \%)$ por daños materiales en la escuela, considera los efectos de depresión, suicidio e idea de suicidio, y rechazo como sinónimo de bullying. ${ }^{4}$ Espinoza Godoy, en su artículo ¿Cómo afecta la violencia en la familia?, menciona que los jovencitos que son sometidos continuamente a situaciones de agresiones presentan un debilitamiento gradual de sus defensas, tanto físicas como psicológicas, lo cual se traduce en un riesgo de enfermedades psicosomáticas, depresión o angustia. ${ }^{6}$

Los hallazgos de la investigación permiten concluir que el $59 \%$ de estudiantes no experimentó violencia intrafamiliar (VIF-), el 34 $\%$ tienen riesgo de violencia, y el $7 \%$ experimentó violencia $(\mathrm{VIF}+)$. La mayor cantidad de estudiantes experimentó violencia psicológica (109 casos). El 3er año registró los más altos porcentajes en los diversos tipos de violencia: física $59 \%$, psicológica $41 \%$, sexual $33 \%$, socio ambiental $38 \%$, económica $33 \%$ y por negligencia $50 \%$, en comparación con los demás años (2do, 4 to y 5 to año) que registraron porcentajes no menos significativos en todos los tipos de violencia. Contrariamente, se observa que los estudiantes del 1er año sufrieron violencia física $9 \%$, psicológica $18 \%$, sexual 3 $\%$ y por negligencia $8 \%$, pero no violencia socio ambiental ni económica. En la dimensión física, se registran cicatrices (52\%), seguido de aparición de enuresis (35\%). En la dimensión de violencia psicológica, el $26 \%$ registró 
depresión y riesgo de depresión, el 24 \% intentó suicidarse y/o tuvo idea suicida, el $15 \%$ tiene riesgo de sufrir psicosis y el $13 \%$ sufre de angustia, en menores porcentajes se evidencia adicción a bebidas alcohólicas, a pastillas para dormir, y a droga. En la dimensión sexual, el 39 $\%$ tenía conductas sexuales inapropiadas (relaciones sexuales con varias parejas en diversos tiempos, inicio temprano de actividad sexual, ver pornografía, bromas con contenido sexual), el $22 \%$ sufrió tocamientos, el $12 \%$ sufrió violación, el $10 \%$ tuvo lesiones genitales, el $7 \%$ tuvo aborto. En la dimensión de violencia social ambiental - intrafamiliar, el $34 \%$ refirió tener buenas relaciones familiares, el $11 \%$ regular y el $3 \%$ malas relaciones familiares. Respecto a sus relaciones personales con otros, el $43 \%$ registró buena integración con otros y el $10 \%$ regular, no registraron malas relaciones con otros. En la dimensión violencia económica, el $75 \%$ refirió dependencia económica de padres; a su vez, el $13 \%$ refirió trabajar con padres, el $9 \%$ se autosostenía, y el $2 \%$ dependía económicamente de otro familiar. En la dimensión violencia por negligencia, el 50 $\%$ reconoció que no tenía atención de salud (padres no se ocuparon de cubrirlos con un seguro), el $33 \%$ incumplía tareas universitarias por falta de economía y el $17 \%$ refirió tener fatiga, sueño y hambre. Entre los efectos de la violencia en la salud mental de los estudiantes tenemos: la depresión con $50 \%$ en 1ro, en 2 do con $37 \%$, en 3 ro con $33 \%$, y en 5 to con $38 \%$. En 4 to año el efecto fue conducta sexual inapropiada con $35 \%$; el intento de suicidio en 1 ro con $11 \%$, en 2 do con el $9 \%$, en 3 ro con el 28 $\%$, en 4 to con el $25 \%$ y en 5 to con el $24 \%$.

Finalmente, recomendamos dar a conocer los resultados a las autoridades de la ESEN para implementar el trato de este tema en tutoría, asesoría y referencia a psicología, para el seguimiento de casos según corresponda, en tanto se implemente el centro de salud mental comunitario universitario. Asimismo, proponemos un proyecto de calidad y estilos de vida saludable dirigido a todos los estudiantes, dentro de responsabilidad social universitaria intramuros. A mediano plazo, se volverá a aplicar el mismo instrumento para monitorear el comportamiento del problema y medir la eficacia de los proyectos.

\section{Referencias}

1. Instituto Nacional de Estadística. INEI Censos Nacionales: perfil sociodemográfico del Perú: [Internet]. http://censos.inei.gob.pe/Anexos/Libro.pdf

2. Organización Mundial de la Salud. Salud Mental del adolescente. UNODC. World Drug Report 2018.

3. Federación Mundial de la Salud Mental. La importancia de cuidar la salud mental de los jóvenes. Informe de la Infocop [internet]. España. 2018

http://www.infocop.es/view_article.asp?id=7 707

4. Chavez-Ayala R. Violencia y salud mental asociados a pensar o haber intentado emigrarinternacionalmente $p \quad 0 \quad r$ adolescentes mexicanos. Cad. Saúde Pública. 2017; 33(6):e00119516. https://doi.org/10.1590/0102-311x00119516

5. Fernández-Montesinos, F. La violencia y el ser humano. Instituto español de estudios estratégicos ieee.es. Boletín 32/2015. p 118.

http://www.ieee.es/Galerias/fichero/docs_an alisis/2015/DIEEEA32-

2015_ViolenciaySerHumano_FAFM.pdf

6. Espinoza Godoy, I. ¿Cómo afecta la violencia en la familia? El siglo de Durango. 2015, 15. p 2.

https://www.elsiglodedurango.com.mx/notici a/579 430.como-afecta-la-violencia-en-lafamilia.html

7. Varillas Suarez, I. Violencia familiar y violencia contra la mujer. 2015 [internet]. http://l.exam-10.com/pravo/12470/index. html

8. Cutipé Y. Población universitaria del Perú sufre de impactos en su salud mental por presión académica. Perú. 2018. https://vital.rpp.pe/vivir-bien/hasta-30-de-lapoblacion-universitaria-del-peru-sufre-deimpactos-en-su-salud-mental-por-presionacademica-noticia-1151266

9. Programa Nacional Contra la Violencia Familiar y Sexual. Impacto y 
Consecuencias de la Violencia contra las Mujeres. Perú.

2016.

http://redin.pncvfs.gob.pe/images/publica cion $2 / i m p a c t o-y$-consecuenciasviolencia.pdf

10. Cueva G. Violencia y adicciones: problemas de salud pública. Revista Peruana de Medicina experimental y $\mathrm{S}$ a I u d $\mathrm{P}$ ú b I i c a . 2012 , 29(1).

https://doi.org/10.17843/rpmesp.2012.29 1.315

11. Castillo JE. Comentarios a la Nueva Ley de violencia contra las mujeres y los integrantes del grupo familiar. UBI LEX ASESORES S.A.C.2016. 403 p. ISBN: 978-612-46900-7-5

12. Ministerio de la Mujer y Poblaciones Vulnerables. Lineamientos Éticos para las investigaciones en Violencia Familiar y Sexual. 2014: 1617 .

https://www.mimp.gob.pe/files/transpare ncia/resoluciones_ministeriales/anexo_r m_338_2014-lineamientos.pdf

13. Ministerio de salud. Prioridades regionales de Investigación en Salud Región Tacna https://web.ins.gob.pe/sites/default/files/ PEI\%202 011-2016\%20Informe\%20 General\%20Anual\%202016.pdf

14. Santillán ML. La violencia afecta la integridad del individuo y de la sociedad. Revista Ciencia UNAM. 06-09-2013. http://ciencia.unam.mx/leer/252/La_viole ncia_afecta_la_integridad_del_individuo y_de_la_sociedad

15. González LA. El problema de violencia y sus enfoques .

16. https://www.alainet.org/es/articulo/181403 https://mariangelesalvarez.com/igualdad/ relacion-de-control-o-igual/laindefension-aprendida/

\section{Correspondencia:}

jennymendoza1960@gmail.com
17. Guía de intervención mhGAP - IRIS PAHO Home

http://iris.paho.org/iris. paho.org $x$ xmlui bit stream $>$ h andle $>9789241549790-$ spa violencia-la-mujer/

18. Aiquipa Tello J. Dependencia emocional en mujeres víctimas de violencia de pareja. Revista de Psicología. 2015, 33 (2):412-437. ISSN 0254-

9247 .

http://www.scielo.org.pe/pdf/psico/v33n2/ a07v33n2.pdf

19. Amor, P y Echeburúa E. Claves Psicosociales para la Permanencia de la Víctima en una Relación de Maltrato. $2010,1(2): 97$ -

104.

https://www.revistaclinicacontemporanea .org/archivos/cc2010v1n2a3.pdf

20. Galvis Lopes M. Teorías y Modelos de Enfermería usados en la Enfermería Psiquiátrica. Rev cuid. 2015, 6(2): 110820.

http://www.scielo.org.co/pdf/cuid/v6n2/v6 n2a12.pdf

21.http://www.cverdad.org.pe/ifinal/pdf/TOMO \%20VI/SECCION\%20CUARTACrimenes $\% 20 y \% 20$ violaciones $\% 20$ DDHH/FINAL AGOSTO/1.5.VIOLENCIA\%20SEXUAL \%20CONTRA\%20LA\% 20MUJER.pdf

22. República del Perú. Constitución Política del Perú. 1993.

peruanohttp://www4.congreso.gob.pe/ntl ey/lmagenes/Constitu/Cons1993.pdf

23. Núñez M, Waldo $F$ y Castillo Soltero M D $\mathrm{P}$, "Violencia Familiar, comentarios a la Ley $\mathrm{N}^{\circ}$ 29282. Doctrina, legislación, jurisprudencia y modelos. Lima, Editorial Ediciones Legales. 2015: 59-60.

24. Ley $\mathrm{N}^{\circ} 29430$, Ley que modifica la ley 27942, Ley de prevención y sanción del hostigamiento sexual. Artículo 4, acápite 4.1 .

Fecha de recepción: 25 de marzo de 2020 Fecha de aceptación: 05 de julio 2020 